\title{
Publisher Correction to: Three-dimensional tumor growth in time-varying chemical fields: a modeling framework and theoretical study
}

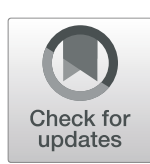

Markos Antonopoulos*, Dimitra Dionysiou, Georgios Stamatakos and Nikolaos Uzunoglu

\section{Correction to: BMC Bioinformatics \\ https://doi.org/10.1186/s12859-019-2997-9}

Following publication of the original article [1], the authors noticed that the following errors were introduced by $\mathrm{pdf} / \mathrm{html}$ formatting issues. The original article has been corrected. The publisher apologizes to the authors and readers for these errors.

\section{Page 9, first column:}

The paragraph

“- $o b_{t}(A)$ : sec) by the local vascular network within/sec) by the local vascular network within $A$ during the previous time interval $t-\Delta \tau \rightarrow t$ "

Should be replaced with

“- $o_{-} b_{t}(A)$ : Oxygen supply rate (pmols/sec) by the local vascular network within $A$ during the previous time interval $t-\Delta \tau \rightarrow t$ "

In the subsequent paragraph, i.e. the paragraph starting starting with the phrase " $-g l_{-} b_{t}(A)$ : Glucose supply rate ..." ,

the phrase " interval- $-\Delta \tau \rightarrow t$ " should be replaced with "interval $t-\Delta \tau \rightarrow t$ "

\section{Page 11, second column:}

The equation $\beta \geq 1-\frac{6 O_{a v}}{l_{t}(A) K_{A T P} \Delta \tau} \equiv \beta_{-}$should be $\beta \geq 1$ $\frac{6 O_{a v}}{l_{t}(A) K_{A T P} \Delta \tau} \equiv \underline{\beta}$.

In the subsequent sentence, i.e. "Since $a_{o}(\beta)$ is increasing, $\beta_{-}$is actually ..." the " $\beta_{-}$" should be " $\beta$ ".

In the subsequent paragraph (first bullet point), in the sentence "If $\beta_{-}>\beta_{2}$, we have that ... " the " $\beta_{-}$" should be " $\beta$ ".

In the subsequent paragraph (second bullet point), i.e. "If $\beta_{-} \leq \beta_{2}$, we have that for each $\beta \in\left[\max \left(\beta_{-}, \beta_{1}\right), \beta_{2}\right]$ it holds that $a_{o}(\beta) \geq 0$. " all occurences of " $\beta_{-}$" should be " $\underline{\beta}$ ".

\footnotetext{
* Correspondence: markosan@central.ntua.gr

Institute of Communication and Computer Systems, National Technical University of Athens, Athens, Greece
}

In paragraph 11a, first line, " $a_{o}(\beta)$ " should be " $a_{g l}(\beta)$ ”. Page 12, first column:

In the sentence " Case 2.2.1. If $\beta>\beta_{2}$ or $\bar{\beta}<\beta_{1}$ or $\min \left(\bar{\beta}, \beta_{2}\right)<\max \left(\beta_{-}, \beta_{1}\right)$, the analysis above implies that $\ldots$ " the inequality $\beta>\beta_{2}$ should be $\beta>\beta_{2}$ and the inequality $\min \left(\bar{\beta}, \beta_{2}\right)<\max \left(\beta_{-}, \beta_{1}\right)$ should be $\min (\bar{\beta}$, $\left.\beta_{2}\right)<\max \left(\beta, \beta_{1}\right)$.

In the sentence " Case 2.2.2. If $\beta \leq \beta_{2}, \bar{\beta} \geq \beta_{1}$ and $\min (\bar{\beta}$, $\left.\beta_{2}\right) \geq \max \left(\beta_{-}, \beta_{1}\right) \ldots$ " the inequality $\beta \leq \beta_{2}$ should be $\beta \leq \beta_{2}$ and the inequality $\min \left(\bar{\beta}, \beta_{2}\right) \geq \max \left(\beta_{-}, \beta_{1}\right)$ should be $\min \left(\bar{\beta}, \beta_{2}\right) \geq \max \left(\beta, \beta_{1}\right)$.

In the same paragraph, the mathematical expression $\beta \in\left[\max \left(\beta_{-}, \beta_{1}\right), \min \left(\bar{\beta}, \beta_{2}\right)\right]$ should be $\beta \in\left[\max \left(\beta, \beta_{1}\right)\right.$, $\left.\min \left(\bar{\beta}, \beta_{2}\right)\right]$

\section{Page 12, second column:}

In the sentence " Again, we pick a random $\tilde{\beta}$ in $\left[\max \left(\beta_{-}\right.\right.$, $\left.\left.\beta_{1}\right), \min \left(\bar{\beta}, \beta_{2}\right)\right]$. " the mathematical expression $\left[\max \left(\beta_{-}\right.\right.$, $\left.\left.\beta_{1}\right), \min \left(\bar{\beta}, \beta_{2}\right)\right]$ should be $\left[\max \left(\underline{\beta}, \beta_{1}\right), \min \left(\bar{\beta}, \beta_{2}\right)\right]$

\section{Page 14, second column:}

The equations

$$
\begin{aligned}
o b \max _{t+\Delta \tau}(A)= & \left(1-f_{r}\left(l_{t}(A), n c_{t}(A)\right) v_{r}\right. \\
& +s w_{t}(A) f_{e}\left(l_{t}(A), n c_{t}(A),\right. \\
& \left.\left.n n_{t}(A)\right) v_{e}\right) \cdot o b \max _{t}(A) \\
g l b \max _{t+\Delta \tau}(A)= & \left(1-f_{r}\left(l_{t}(A), n c_{t}(A)\right) v_{r}\right. \\
& +s w_{t}(A) f_{e}\left(l_{t}(A), n c_{t}(A),\right. \\
& \left.\left.n n_{t}(A)\right) v_{e}\right) \cdot g l b \max _{t}(A)
\end{aligned}
$$


should be

$$
\begin{aligned}
o_{-} b_{-} \max _{t+\Delta \tau}(A)= & \left(1-f_{r}\left(l_{t}(A), n c_{t}(A)\right) v_{r}\right. \\
& +s w_{t}(A) f_{e}\left(l_{t}(A), n c_{t}(A),\right. \\
& \left.\left.n n_{t}(A)\right) v_{e}\right) \cdot o_{-} b_{-} \max _{t}(A) \\
g l_{\_} b_{-} \max _{t+\Delta \tau}(A)= & \left(1-f_{r}\left(l_{t}(A), n c_{t}(A)\right) v_{r}\right. \\
& +s w_{t}(A) f_{e}\left(l_{t}(A), n c_{t}(A),\right. \\
& \left.\left.n n_{t}(A)\right) v_{e}\right) \cdot g l_{-} b_{-} \max _{t}(A) .
\end{aligned}
$$

The equations

$$
\begin{aligned}
o b \max _{t+\Delta \tau}(A)= & \left(1-f_{r}\left(l_{t}(A), n c_{t}(A)\right) r_{3} v_{r}\right. \\
& +s w_{t}(A) f_{e}\left(l_{t}(A), n c_{t}(A),\right. \\
& \left.\left.n n_{t}(A)\right) r_{4} v_{e}\right) \cdot o b \max _{t}(A) \\
g l b \max _{t+\Delta \tau}(A)= & \left(1-f_{r}\left(l_{t}(A), n c_{t}(A)\right) r_{3} v_{r}\right. \\
& +s w_{t}(A) f_{e}\left(l_{t}(A), n c_{t}(A),\right. \\
& \left.\left.n n_{t}(A)\right) r_{4} v_{e}\right) \cdot g l b \max _{t}(A)
\end{aligned}
$$

should be

$$
\begin{aligned}
o \_b \_\max _{t+\Delta \tau}(A)= & \left(1-f_{r}\left(l_{t}(A), n c_{t}(A)\right) r_{3} v_{r}\right. \\
& +s w_{t}(A) f_{e}\left(l_{t}(A), n c_{t}(A),\right. \\
& \left.\left.n n_{t}(A)\right) r_{4} v_{e}\right) \cdot o \_b \_\max _{t}(A) \\
g l \_b \_\max _{t+\Delta \tau}(A)= & \left(1-f_{r}\left(l_{t}(A), n c_{t}(A)\right) r_{3} v_{r}\right. \\
& +s w_{t}(A) f_{e}\left(l_{t}(A), n c_{t}(A),\right. \\
& \left.\left.n n_{t}(A)\right) r_{4} v_{e}\right) \cdot g l_{-} b_{-} \max _{t}(A)
\end{aligned}
$$

\section{Page 15, first column:}

The equations

$$
\begin{aligned}
o b \max _{t+\Delta \tau}(A)= & \left(1-\frac{l_{t}(A)+n c_{t}(A)}{M} r_{3} v_{r}\right. \\
& \left.+s w_{t}(A) \frac{M-l_{t}(A)-n c_{t}(A)-n n_{t}(A)}{M} r_{4} v_{e}\right) \\
& . o b \max _{t}(A)
\end{aligned}
$$$$
g l b \max _{t+\Delta \tau}(A)=\left(1-\frac{l_{t}(A)+n c_{t}(A)}{M} r_{3} v_{r}\right.
$$$$
\left.+s w_{t}(A) \frac{M-l_{t}(A)-n c_{t}(A)-n n_{t}(A)}{M} r_{4} v_{e}\right)
$$$$
\cdot g l b \max _{t}(A)
$$

should be

$$
\begin{aligned}
o_{-} b_{-} \max _{t+\Delta \tau}(A)= & \left(1-\frac{l_{t}(A)+n c_{t}(A)}{M} r_{3} v_{r}\right. \\
& \left.+s w_{t}(A) \frac{M-l_{t}(A)-n c_{t}(A)-n n_{t}(A)}{M} r_{4} v_{e}\right) \\
& \cdot o \_b_{-} \max _{t}(A) \\
g l \_b \_\max _{t+\Delta \tau}(A)= & \left(1-\frac{l_{t}(A)+n c_{t}(A)}{M} r_{3} v_{r}\right. \\
& \left.+s w_{t}(A) \frac{M-l_{t}(A)-n c_{t}(A)-n n_{t}(A)}{M} r_{4} v_{e}\right) \\
& . g l_{\_} b_{-} \max _{t}(A)
\end{aligned}
$$

\section{Page 15, second column:}

The equations

$$
\begin{aligned}
o b \max _{t+\Delta \tau}(A)= & \left(1-\frac{l_{t}(A)+n c_{t}(A)}{M} r_{3} v_{r}\right. \\
+ & \left.s w_{t}(A) \frac{M-l_{t}(A)-n c_{t}(A)-n n_{t}(A)}{M} r_{4} v_{e}\right) \\
& . o b \max _{t}(A) \\
g l b \max _{t+\Delta \tau}(A)= & \left(1-\frac{l_{t}(A)+n c_{t}(A)}{M} r_{3} v_{r}\right. \\
& \left.+s w_{t}(A) \frac{M-l_{t}(A)-n c_{t}(A)-n n_{t}(A)}{M} r_{4} v_{e}\right) \\
& . g l b \max _{t}(A)
\end{aligned}
$$

Should be

$$
\begin{aligned}
o_{-} b_{-} \max _{t+\Delta \tau}(A)= & \left(1-\frac{l_{t}(A)+n c_{t}(A)}{M} r_{3} v_{r}\right. \\
+ & \left.s w_{t}(A) \frac{M-l_{t}(A)-n c_{t}(A)-n n_{t}(A)}{M} r_{4} v_{e}\right) \\
& \cdot o_{-} b_{-} \max _{t}(A) \\
g l \_b \_\max _{t+\Delta \tau}(A)= & \left(1-\frac{l_{t}(A)+n c_{t}(A)}{M} r_{3} v_{r}\right. \\
& \left.+s w_{t}(A) \frac{M-l_{t}(A)-n c_{t}(A)-n n_{t}(A)}{M} r_{4} v_{e}\right) \\
& . g l_{-} b_{-} \max _{t}(A)
\end{aligned}
$$

The second equation appearing in this column, i.e.

$$
\begin{aligned}
g l_{-} b_{t+\Delta \tau}(A) & =B_{o_{-} b_{-} \max _{t+\Delta \tau}(A)}\left(g l_{-} b_{t}(A)\right. \\
& \left.+r_{2}\left(\left(\overline{g l_{0}}-g l_{t}(A)\right) / \Delta \tau\right)\right)
\end{aligned}
$$

Should be

$$
\begin{aligned}
g l \_b_{t+\Delta \tau}(A)= & B_{g l \_b-} \max _{t+\Delta \tau}(A)\left(g l_{-} b_{t}(A)\right. \\
& \left.+r_{2}\left(\left(\overline{g l_{0}}-g l_{t}(A)\right) / \Delta \tau\right)\right)
\end{aligned}
$$

Published online: 16 October 2019

\section{Reference}

1. Antonopoulos M, Dionysiou D, Stamatakos G, Uzunoglu N. Threedimensional tumor growth in time-varying chemical fields: a modeling framework and theoretical study. BMC Bioinformatics. 2019;20:Article number: 442. 